Case Report

\title{
Laparoscopic M arsupialization of a Giant Non-Parasitic Splenic Cyst (Npsc) in the Paediatric Age Group
}

\author{
Amol Dilip Amonkar ${ }^{1}$, Raghushankar ${ }^{2}$, Sandeep Rai ${ }^{3}$ Sattar Khan ${ }^{4} \&$ Ganesh G. V. ${ }^{5}$ \\ ${ }^{1,4.5}$ Post graduates, ${ }^{2}$ Associate professor, ${ }^{3}$ Professor and HOD, Department of Paediatric Surgery, \\ K.S. Hegde M edical Academy, Nitte University, M angalore - 575 018, Karnataka, India.
}

\author{
Correspondence \\ Amol Amonkar \\ Post graduate, Department of Surgery, K. S. Hegde Medical Academy, Nitte University, M angalore - 575 018, Karnataka, India. \\ Mobile : +919886535829 E-mail : amonkaramol@gmail.com
}

\begin{abstract}
Nonparasitic splenic cysts are rare clinical lesions of the spleen. The management has entailed partial or total splenectomy via an open approach. Recently, laparoscopic approaches have been developed. In this report, we describe laparoscopic marsupialization of a giant splenic cyst (diameter $>15 \mathrm{~cm}$ ).A 15-year-old Indian child presented with a four and a half year history of mass in upper left quadrant associated with left upper quadrant pain which increased since 2 days. Physical examination revealed a large, nontender left upper quadrant mass with minimal movement on respiration. Computed tomography scan confirmed a cyst arising from the spleen, measuring $20 \times 15 \mathrm{~cm}$ suggestive of a primary splenic cyst. Echinococcus and Entamoeba histolytica serologies were negative. Laparoscopic exploration was performed. three liters of brown fluid were aspirated and intraoperative cytology confirmed a nonparasitic cyst. The cyst wall was excised, preserving the spleen. The patient's recovery was uneventful, and he was discharged, tolerating a regular diet on postoperative day 3 . At follow-up, the patient was asymptomatic and showed no evidence of recurrence. Non-parasitic splenic cysts are rare lesions. Laparoscopic marsupialization is safe and effective and should be considered the treatment of choice even for giant splenic cyst.
\end{abstract}

Key words: laparoscopic, marsupialization, giant splenic cyst

\section{Introduction}

Splenic cysts are rare lesions and should be suspected when a mass is noted in the left upper quadrant [1].The traditional classification divides them into true cysts (primary) and pseudo cysts (secondary) on the basis of presence or absence of an epithelial lining.[1].Primary cysts can be divided into those with parasitic and those with non-parasitic causes. Secondary cysts are usually seen following abdominal trauma and are thought to be a late complication of an intrasplenic haematoma, such cysts may become quite large and cysts greater than $15 \mathrm{cms}$ are considered as giant cysts.[1]Formerly the treatment of choice for splenic cysts Access this article online Quick Response Code

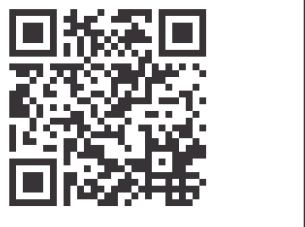
consisted of total splenectomy via an open approach but however due to a small but real risk of overwhelming post splenectomy sepsis (OPSI), spleen sparing techniques have been developed, additionally advances in minimally invasive surgery have resulted in effective treatment with less morbidity. Although laparoscopic hemi-splenectomy has been reported, most non - parasitic splenic cysts (NPSC) may be unroofed or marsupialized with good results. In this paper, we present a giant splenic cyst which was successfully treated by laparoscopic marsupialization [1].

\section{Case Report}

A 15 year old child presented with a mass in the upper abdomen which was insidious in onset and gradually progressed to its present size over 4 and half years associated with a dull aching type of pain in the left upper quadrant of the abdomen which increased since 2 days. Physical examination revealed a large firm, fixed, on non tender mass measured $20 \times 15 \mathrm{~cm}$ in the left upper quadrant, the rest of the physical examination was unremarkable. An ultrasound abdomen showed a 20 X15 $\mathrm{cm}$ cystic lesion arising from the spleen. The computed tomography (CT) scan of the abdomen confirmed a large 
$20 \times 15 \mathrm{~cm}$ cystic lesion arising from the spleen exerting a significant mass effect on the surrounding organs, the stomach, duodenum and pancreas were displaced medially and the left kidney was compressed posteriorly[Fig 1 and 2]. Because of the symptoms and size of the cyst, surgery was recommended and the patient agreed to proceed. After induction of general anaesthesia, the patient was placed in the supine position, after establishing pneumoperitoneum, a $10 \mathrm{~mm} 30^{\circ}$ laparoscope was inserted through a right iliac fossa port, the abdomen was explored and a giant splenic cyst was identified [Fig 3].The mass displaced the transverse colon inferiorly and the stomach and duodenum medially. Two additional $5 \mathrm{~mm}$ trocars were placed in the right lumbar and left lower abdominal quadrants. After delineating the cyst wall all around. Three litres of fluid were removed before the cyst was completely decompressed. Intra-operative fluid serologies were negative for Echinococcus and
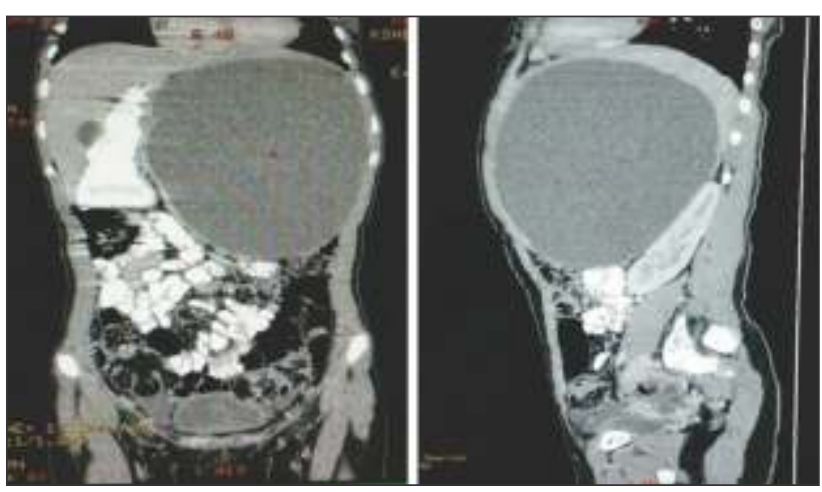

Fig. 1a and b : Computed tomography (CT) showing a large 20X15 $\mathrm{cm}$ cystic lesion arising from the spleen exerting a significant mass effect on the surrounding organs, stomach, duodenum and pancreas were displaced medially and the left kidney was compressed posteriorly.

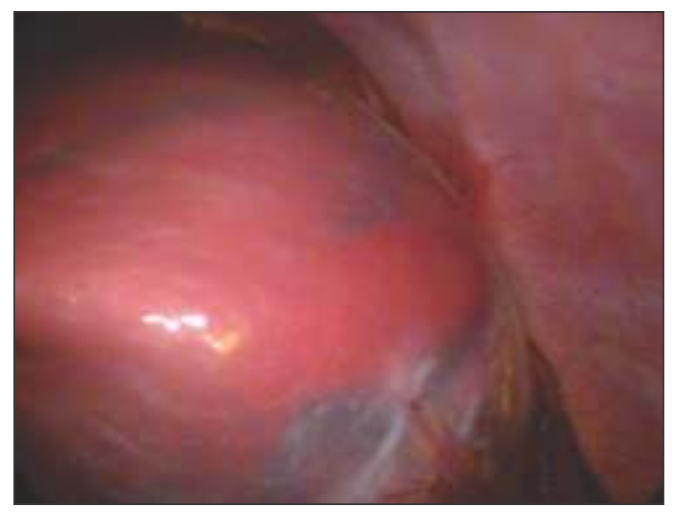

Fig. 2 : Laparoscopic view of the giant splenic cyst.
Entamoeba histolytica. The cyst was then widely unroofed by excising the entire posterior and lateral wall [Fig 4]. After deroofing, the trabecular cyst lining was visualized and debris was removed, thus complete marsupialization was achieved [Fig 5]. The cyst wall was placed in a specimen bag and relieved through the $10 \mathrm{~mm}$ port site [Fig 6]. Postoperative period was uneventful. Histopathology was suggestive of an primary epithelial cyst of the spleen.

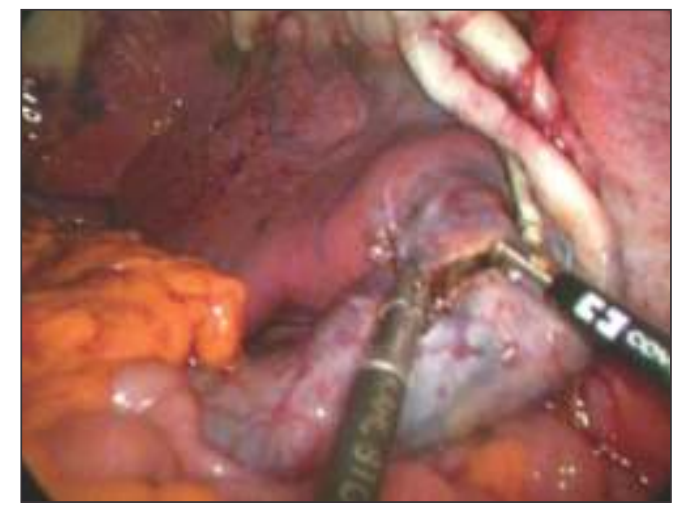

Fig. 3 : Laparoscopic view of the decompressed splenic cyst

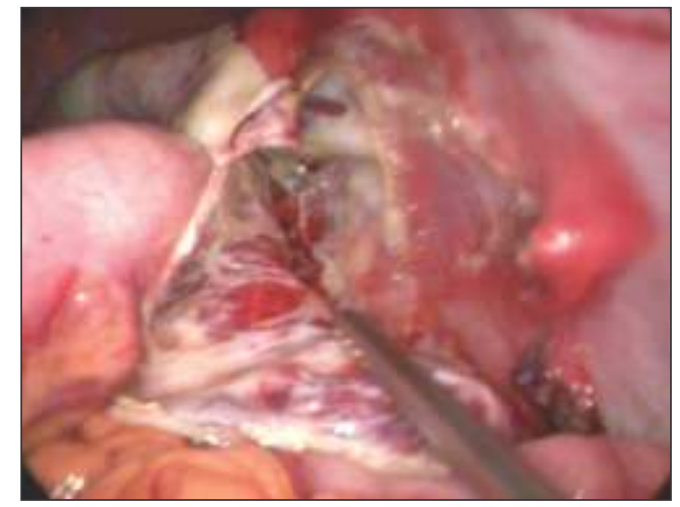

Fig. 4 : Laparoscopic view of the cystic cavity and debris

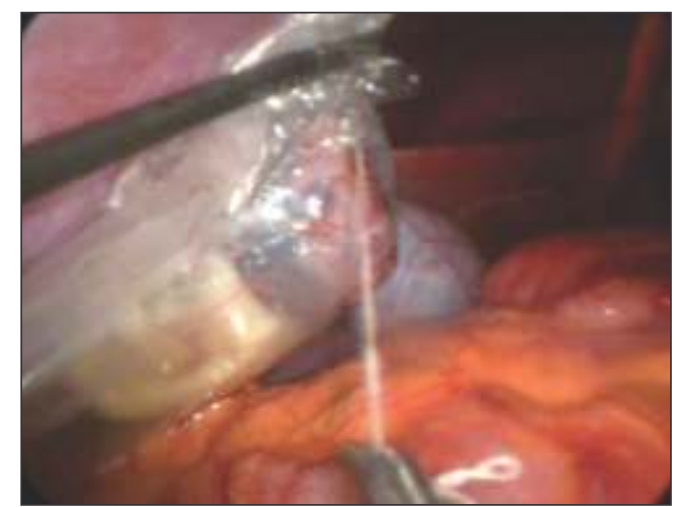

Fig. 5 : Laparoscopic view of the cyst wall was placed in a specimen bag. 


\section{Discussion}

In 1790, Berthelot described the first echinococcal splenic cyst and in 1829 and Andral described the first NPSC [1], since then approximately 800 cases of NPSC have been reported and a recent increase has occurred in the incidence of post traumatic cyst.[2]. Several classifications for splenic cysts exist, the most widely adopted is that of Mcclure and Altemeirer which divides splenic cysts into two groups. A specific secreting membrane lines primary or true cyst, the lining may be epithelial, endothelial or parasitic. Echinococcus is the most common parasitic cause and most of the other primary cysts are congenital. False or secondary cysts do not have a secretory lining and may be serous, inflammatory, degenerative or haemorrhagic .Blunt abdominal trauma with occult injury to the spleen is the most common cause for secondary cyst [3].Patients with a suspected splenic cyst may have a palpable mass on physical examination ,but often do not. Other causes of splenomegaly including myeloid metaplasia, haemolytic anemia, mononucleosis and portal hypertension must be excluded [4]. Ultrasound and CT imaging may help to distinguish cystic from solid lesion and to characterize cyst loculations [5]. Although the natural history of secondary splenic cysts is not completely known, a risk of rupture, infection or haemorrhage. Asymptomatic patients with small cysts $(5 \mathrm{cms})$ may be observed and usually do not require treatment .For symptomatic patients and for large cysts ( $>5 \mathrm{cms}$ ), surgical treatment is indicated. Historically,

\section{References}

1. Sierra R, Brunner W C et al; laparoscopic marsupialization of a giant post - traumatic splenic cyst.J SLS.2004 oct-dec, 8(4):384-388.

2. Pachter HL, Hofstetter SR et al. Traumatic cyst of thye spleen -the role of cystectomy and splenic preservation; experience with seven consecutive patients J Trauma 1993;35:430-436

3. Knudson $P$, Coon W, Schnitzer B, Leipman M, Arbon A splenomegaly without an apparent cause. Surg,Gynec,Obstet 1982;155:705-708.

4. Cinzia L, Konstantinos NH et al .post traumatic cyst of the spleen :a case report and review of literature: Int surg 2002;87:152-156.

5. Van der zee DC, Kramen WL, Ure BM et al.Iaparoscopic management of a large post-traumatic splenic cyst.Surg Endosc. 1999; 13:1241-1242 the treatment of choice for NPSCs has been total splenectomy. Although splenectomy may be indicated for patients with patients with parasitic cysts, spleen sparing techniques have evolved as the treatment of choice for NPSCS so that the risk of OPSI may be avoided [1]. The incidence of OPSI is $3.3 \%$ to $4.4 \%$ among children and $0.9 \%$ to $3.2 \%$ in adults with mortality ranging from 0.8 to $4.4 \%$ [1].The organism most frequently associated with OPSI are Streptococcus pneumoniae, Neisseria meningitides, Escherichia coli and Haemophilus influenza. Spleen sparing techniques such as percutaneous drainage with or without injection of a sclerosing agent have been described but results in a high rate of recurrence [1].On the other hand ,partial splenectomy and marsupialization via an open approach has been proven effective [1].Laparoscopic approaches for both techniques have recently proven feasible and result in less operative morbidity. In our patient, a partial splenectomy was unnecessary because the cyst was broad -based and amendble for unroofing. For deeply seated, narrow based cysts, a hemisplenctomy may be indicated to prevent recurrence.

\section{Conclusion}

Non parasitic splenic cysts are rare lesions but may be associated with significant symptoms. Laparoscopic marsupialization is safe and effective and feasible for giant splenic cysts, a laparoscopic spleen sparing technique should be considered the treatment of choice. 